\title{
IL-33 is overexpressed in the inflamed arteries of patients with giant cell arteritis
}

\author{
Francesco Ciccia, ${ }^{1}$ Riccardo Alessandro, ${ }^{2}$ Aroldo Rizzo, ${ }^{3}$ Stefania Raimondo, ${ }^{2}$ \\ AnnaRita Giardina, ${ }^{1}$ Francesca Raiata, ${ }^{3}$ Luigi Boiardi, ${ }^{4}$ Alberto Cavazza, ${ }^{4}$ \\ Giuliana Guggino, ${ }^{1}$ Giacomo De Leo, ${ }^{2}$ Carlo Salvarani, ${ }^{4}$ Giovanni Triolo ${ }^{1}$
}

${ }^{1}$ Dipartimento Biomedico di

Medicina Interna e

Specialistica, Sezione di

Reumatologia, Università di

Palermo, Palermo, Italy

${ }^{2}$ Dipartimento di Biopatologia e

Biotecnologie Mediche e

Forensi, Università di Palermo,

Palermo, Italy

${ }^{3}$ Unità Operativa di Anatomia

Patologica, Azienda

Ospedaliera Ospedali Riuniti

'Villa Sofia-Cervello', Palermo,

Italy

${ }^{4}$ Unità operativa di

Reumatologia, Azienda

Ospedaliera Arcispedale

S. Maria Nuova, Reggio Emilia,

Italy

\section{Correspondence to}

Professor Giovanni Triolo, Department of Internal

Medicine, Division of

Rheumatology, Piazza delle

Cliniche 2. Palermo 90127.

Italy; g.triolo@unipa.it

Received 5 January 2012

Accepted 17 July 2012

Published Online First

7 August 2012

\section{ABSTRACT}

Objective To study the expression of interleukin (IL)-33 and to evaluate its relationship with macrophage polarisation in artery biopsy specimens from patients with giant cell arteritis (GCA).

Methods IL-33, ST2, p-STAT-6 and perivascular IL-1 receptor-associated kinase 1 ( $p$-IRAK1) tissue distribution was evaluated by immunohistochemistry. Inducible nitric oxide synthase and CD163 were also used by immunohistochemistry to evaluate the M1 and M2 polarisation, respectively. Quantitative gene expression analysis of IL-33, T-helper (Th)2-related transcription factor STAT6, Th2 cytokines (IL-4, IL-5, IL-25) and interferon (IFN)- $\gamma$ was performed in artery biopsy samples obtained from 20 patients with GCA and 15 controls. Five additional patients who had received prednisone when the temporal artery biopsy was performed were also enrolled.

Results IFN- $\gamma$ and IL-33 were significantly overexpressed in the inflamed arteries of GCA patients. IL-33 overexpression was not accompanied by a concomitant increase of Th2 cytokines. Neovessels scattered through the inflammatory infiltrates were the main sites of IL-33 expression. The expression of IL-33 receptor ST2 and of p-IRAK1 was also increased in GCA patients. Arteries from glucocorticoid-treated patients had a lower expression of IL-33. IL-33 was accompanied by the expression of $\mathrm{p}$-STAT6 and a clear M2 macrophages polarisation.

Conclusions A role for IL-33 in the inflammation of GCA patients is supported by these findings.

\section{INTRODUCTION}

Giant-cell arteritis (GCA) is an inflammatory disease of blood vessels most commonly involving large and medium arteries of the head. ${ }^{1}$ The pathogenesis of the disease is still unknown. Indeed, several studies have demonstrated that both the innate and adaptive immune system contribute to GCA pathogenesis. ${ }^{2}{ }^{3} \mathrm{~T}$ lymphocytes and macrophages in particular seem to be the main actors of inflammation in GCA patients. ${ }^{4}$

The entry of inflammatory cells in artery inflamed walls is mainly due to their interaction with specific adhesion molecules expressed on the inflamed endothelium. In GCA inflammationinduced angiogenesis occurs. Neovessels and advential vasa vasorum are the main sites where adhesion molecules for leucocytes are expressed and, therefore, the main sites through which circulating leucocytes are recruited into the vascular bed. ${ }^{4}$

Interleukin (IL)-33, a member of the IL-1 cytokine family, ${ }^{6}$ is a recently described novel activator of endothelial cells, which promotes adhesion molecules and proinflammatory cytokine expression in the endothelium and angiogenesis and vascular permeability in vitro and in vivo. ${ }^{7-9}$

IL-33 may act both as a traditional cytokine through activation of the IL-1 receptor-related protein ST2 and as an intracellular nuclear factor by translocating to the nucleus, where it binds to the chromatin and modulates gene expression. ${ }^{10-12}$ IL-33 has also potent immune-modulating properties that are mediated by the downstream induction of cytokines and chemokines. Unlike the other IL-1 family members, IL-33 primarily induces T-helper (Th)2 immune responses and the polarisation of macrophages through the alternative pathway of activation (the so-called M2 macrophages). ${ }^{13}$

In GCA all injury mechanisms have been related to effector macrophages. Different pathways of macrophage activation have been described in humans with classically activated M1 macrophages, mainly inducing tissue inflammation and M2 macrophages involved in tissue repair and remodelling. ${ }^{14-16}$ Consistently with Th1 immune response M1 macrophages seem to be mainly involved in the pathogenesis of $\mathrm{GCA}^{45}$ even if the exact contribution of $\mathrm{M} 2$ macrophages has not yet been investigated.

The role of IL-33 in human inflammatory diseases, such as rheumatoid arthritis and psoriasis, has been demonstrated, identifying IL-33 as a potential therapeutic target in chronic inflammation. ${ }^{17-21}$ Whether this cytokine could be implicated in the immune response characteristic of GCA is still unknown.

In the present study we investigated the expression of IL-33 and its relationship with the macrophage polarisation in the inflamed arteries of GCA patients.

\section{METHODS}

\section{Patients}

Twenty consecutive patients ( 15 women, five men) diagnosed as having GCA were studied. Fifteen patients had evidence of classic transmural inflammation while five displayed a small-vessel vasculitis (SVV) surrounding an uninflamed temporal 
artery. These five patients satisfied the American College of Rheumatology 1990 criteria for the classification of GCA. ${ }^{22}$ Furthermore, none of these five patients had clinical manifestations suggesting a systemic vasculitis other than GCA when temporal artery biopsy was performed or during the follow-up period (median 34.5 months).

Their median age was 75 years (range 60-85 years). The erythrocyte sedimentation rate was $74 \mathrm{~mm} / \mathrm{h}$ (range 68-120). Headache and artery tenderness and/or decreased or absent temporal artery pulsation were present in 16 out of 20 patients. At the time of diagnosis no patients were receiving steroids and five patients had vision loss. Polymyalgia rheumatica was associated in five patients.

To assess the effect of glucocorticoids on IL-33 expression we also consecutively selected temporal artery samples from five additional patients with biopsy-confirmed GCA who had received prednisone ( $1 \mathrm{mg} / \mathrm{kg}$ per day) for an average of 21 days (range 8-33) when the temporal artery biopsy was excised.

To evaluate the baseline inflammatory response at the time of diagnosis four parameters (score ranges from 0 to 4) were used: fever, weight loss, erythrocyte sedimentation rate of $85 \mathrm{~mm} / \mathrm{h}$ or greater and haemoglobin less than $11.0 \mathrm{mg} / \mathrm{dl}$.

Fifteen histologically normal temporal artery samples from 15 consecutive patients, matched for sex and age (12 women, three men; median 75.8 years, range 66-85 years) with suspected GCA who had negative biopsy results served as controls. However, to be sure the patients were really negative controls we excluded biopsy-negative patients satisfying American College of Rheumatology 1990 GCA classification criteria ${ }^{22}$ and patients treated with corticosteroids. Furthermore, the control patients had a long follow-up period (median 44 months) and they never developed clinical manifestations related to GCA or other vasculitis. From 11 patients with biopsy-confirmed GCA and five controls, total RNA was extracted from frozen fragments of the temporal artery biopsies excised before starting treatment.

The study was approved by the ethics committee of local institutions. Patients' signed informed consent for the collection and storage of biological material was also obtained.

\section{Histological assessment}

Biopsy specimens were evaluated by two experienced pathologists (AR and $\mathrm{AC}$ ) and classified into three main categories: (1) classic GCA if histological examination of temporal artery biopsies showed transmural infiltration predominantly of lymphomononuclear cells in the temporal artery wall, with or without giant cells; (2) SVV surrounding an uninflamed temporal artery if histological examination of temporal artery biopsies showed aggregates of inflammatory mononuclear cells arranged around capillaries essentially located in the connective tissue around the adventitia and absence of inflammation in temporal artery; $^{23}$ (3) biopsy specimens without evidence of inflammation in temporal artery and in the surrounding connective tissue. Numbers of neovessels distributed within the wall of inflamed arteries were counted and expressed as the median of the number of microvessels in three random high-power microscopic fields (400× magnification).

\section{Immunohistochemical analysis of GCA biopsy samples}

Tissue samples were immediately fixed with $4 \%$ formaldehyde and embedded in paraffin. Immunohistochemistry for IL-33 and ST2 was performed on $5-\mu \mathrm{m}$-thick paraffin-embedded sections from arteries and from tonsils (used as positive controls) as previously described. ${ }^{3}$ The primary antibodies mouse monoclonal anti-human IL-33 (1:200 dilution; Novus Biological, Littleton, Colorado, USA), rabbit polyclonal antihuman ST2 (dilution 1:100; Novus Biological), rabbit antihuman p-STAT6 ( $p$ Tyr 641) (dilution 1:100; Novus Biological), rabbit anti-human perivascular IL-1 receptorassociated kinase 1 (p-IRAK1) (clone $\mathrm{H}-273$ ) (dilution 1:25; Santa Cruz Biotechnology, Mill Valley, California, USA), mouse anti-human CD68 (macrophages) (clone PG-M1) (dilution 1: 200; Dako A/S, Cambridge, UK) and mouse anti-humanCD163 (clone 10D6) (dilution 1:100; Novocastra, Newcastle, UK) were added and incubated for $1 \mathrm{~h}$ at room temperature. Isotype-matched irrelevant antibodies were used as a negative control (mouse IgG1 monoclonal antibody (ab27479) and rabbit IgG polyclonal antibody (ab27472), AbCam, Cambridge, UK). Following three washes with Tris-buffered saline, slides were incubated for $30 \mathrm{~min}$ with peroxidase-conjugated Dako EnVision polymer. After three further washings, peroxidase activity was visualised using diaminobenzidine chromogen (Dako), and slides were lightly counterstained with haematoxylin before dehydration and mounting in DePex (VWR International, Oslo, Norway). The number of IL-33 and CD-163-expressing cells was determined by counting IL-33 immunoreactive cells on photomicrographs obtained from three random high-power microscopic fields (400× magnification) under a Leica DM2000 optical microscope, using a Leica DFC320 digital camera (Leica, Rijswijk, The Netherlands).

RNA extraction from temporal artery biopsies and quantitative TaqMan reverse transcriptase PCR for IFN- $\gamma$, IL-4, IL-5, IL-25, IL-33 and STAT6

Temporal artery biopsies were stored soon after removal in RNAlater solution (Applied Biosystems, Foster City, California, USA). Each sample was lysed in a tissue homogeniser and RNA was extracted using the commercially available illustra RNAspin Mini Isolation Kit (GE Healthcare, Little Chalfont, Bucks, UK), according to the manufacturer's instructions. Total RNA was reverse-transcribed to complementary DNA using the high capacity cDNA reverse transcription kit (Applied Biosystems). Samples were stored at $-20^{\circ} \mathrm{C}$ until use. For quantitative TaqMan real-time PCR, master mix and Taqman gene expression assays for GAPDH control and for interferon (IFN)- $\gamma$ (Hs00989291_m1), IL4 (Hs00166237_m1), IL-5 (Hs00602482 $\mathrm{m} 1)$, IL-25 (Hs03044841_m1), IL33 ${ }^{-}$(Hs01125942_m1) and STAT6 (Hs00598625_m1) were obtained from Applied Biosystems. Samples were run in triplicate using the step-one real-time PCR system (Applied Biosystems). Relative changes in gene expression between control and arteritis samples were determined using the $\Delta \Delta \mathrm{C}_{\mathrm{t}}$ method. Levels of the target transcript were normalised to a GAPDH endogenous control, constantly expressed in both groups $\left(\Delta \mathrm{C}_{t}\right)$. For $\Delta \Delta \mathrm{C}_{t}$ values, additional subtractions were performed between arteritis and control $\Delta \mathrm{C}_{\mathrm{t}}$ values. Final values were expressed as fold of induction.

\section{Statistical analysis}

Statistical analysis of quantitative variables was performed using the Mann-Whitney rank-sum test. Correlation analyses were performed by using the Pearson product moment correlation. $p$ Values less than 0.05 were considered significant.

\section{RESULTS}

Clinical and histological features

Fifteen of 20 patients with untreated GCA had evidence of classic transmural inflammation while five displayed SVV 
surrounding a non-inflamed temporal arteritis or an isolated vasa vasorum vasculitis. All patients treated with steroids displayed transmural inflammation. Of the 15 patients with transmural inflammation one or fewer inflammatory parameters were observed in two patients and two or more inflammatory parameters were observed in 13. Two out of the five patients with SVV had one or fewer inflammatory parameters and the remaining displayed two or more inflammatory parameters.

\section{IL-33 and ST-2 expression in the inflamed arteries of GCA} patients

We evaluated the expression of IL-33 and its receptor ST2 at the protein level and its distribution within the tissue.

Intense IL-33 staining was observed only in inflamed arteries (figure 1A-F). In particular, patients with SVV displayed IL-33 expression among inflammatory mononuclear cells arranged around capillaries located in the connective tissue around the adventitia (figure 1B). In classic transmural inflammation IL-33 immunoreactivity was observed in the context of granulomas and some scattered cells of the inflammatory infiltrates (figure 1C-E). IL-33 expression was also observed in microvessels distributed within the vessel wall of inflamed arteries that we considered as neovessels resulting from inflammationdependent angiogenesis (figure 1F). A weak expression of IL-33 was also observed among vascular smooth muscle cells in both subgroups of patients (figure $1 \mathrm{~B}-\mathrm{C}$ ).

As IL-33 induces endothelial activation and angiogenesis through ST2/TRAF6-mediated endothelial nitric oxide production, ${ }^{13}$ we also evaluated the tissue expression and distribution of ST2 in the arterial wall of patients and controls. As shown in figure 2, ST2 expression was observed only in the inflamed arteries (figure 2A-B). In particular, the endothelial cells of newly formed vessels (figure 2C), some scattered cells of the inflammatory infiltrates (figure 2D) and granulomas (figure 2E) were the main cells positive for ST2 in patients displaying transmural inflammation. Of importance is the observation that the same vessels that overexpress IL-33 are intensely positive for ST-2 (figures $1 \mathrm{~F}$ and $2 \mathrm{C}$ ). Immunoreactivity for ST2 was not clearly observed in temporal arteries with SVV (data not shown). As the signal of IL-33/ST2 is mediated through the IL-1 signalling pathway, ${ }^{24}$ we also studied the expression of IRAK1 to demonstrate the functional relevance of IL-33. As shown in figure 3, p-IRAK1 was overexpressed in the inflamed artery wall of patients with transmural inflammation.

\section{IL-33 mRNA is overexpressed in the inflamed arteries of GCA patients}

We also investigated whether arterial inflammation in GCA patients was characterised by upregulation of IL-33 and Th2 cytokines such as IL-4, IL-5, IL-25 and the Th2-related nuclear transcription factor STAT6. As shown in figure 4A a marked and significant increase in arterial expression of IL-33 transcripts was observed in GCA patients with both transmural inflammation and SVV compared with control artery biopsy specimens $(p<0.001$ and $p<0.05$, respectively). IL-33 expression in GCA patients with transmural inflammation was correlated with the number of inflammatory parameters, as described in the Methods section $\left(r^{2}=0.5663, p=0.0012\right)$ (figure $4 \mathrm{~B}$ ) and with the numbers of neovessels $\left(r^{2}=0.49, p=0.0035\right)$ (figure $4 C$ ). Of relevance, the expression of Th2-related cytokines IL-4, IL-5, IL-25 was absent in the inflamed arteries of GCA patients (data not shown), suggesting a role for IL-33 outside the context of a Th2 response. Interestingly, STAT6 was significantly increased only in the inflamed arteries of patients with transmural inflammation compared to controls $(p<0.001)$ (figure 4D). A trend towards an increase in STAT6 expression was also observed in patients with SVV, although statistical significance was not achieved, probably due to the low number of patients enrolled. The function of STAT molecules is reflected by their phosphorylation status, thus we investigated phospho-STAT-6
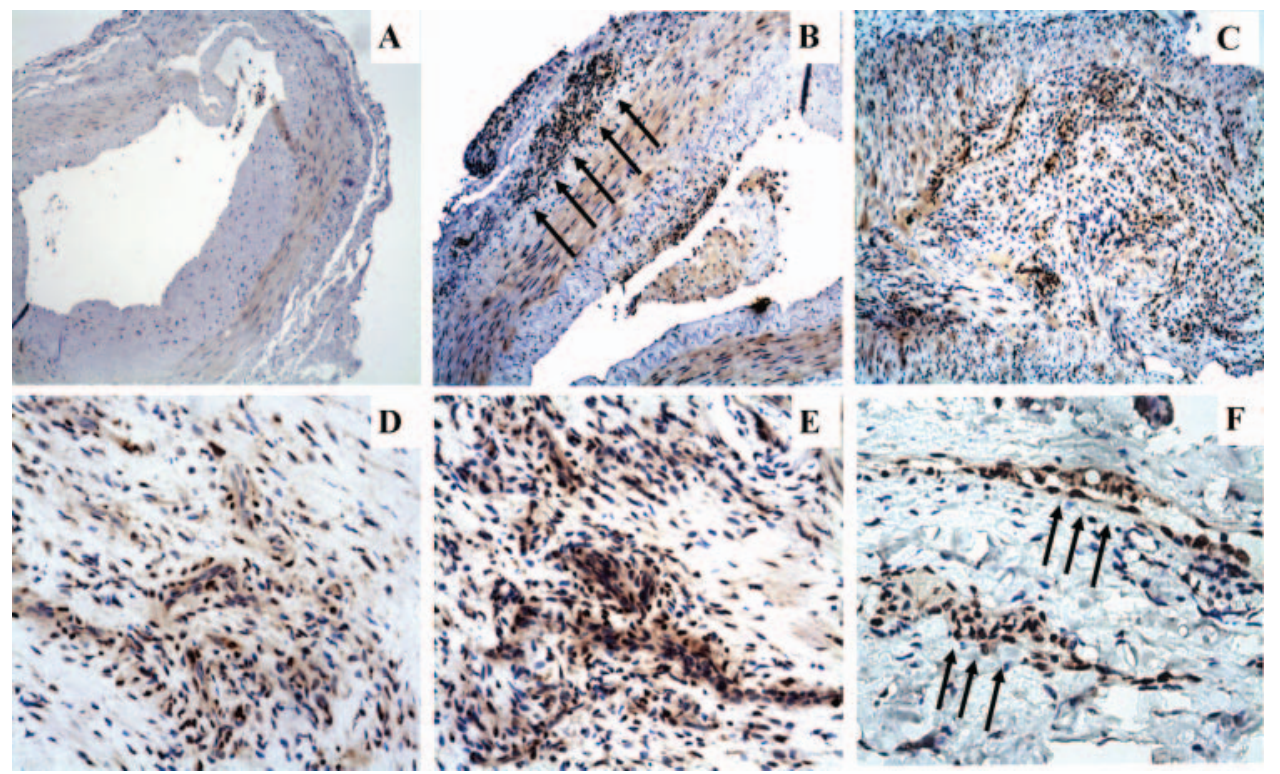

Figure 1 Interleukin (IL)-33 expression in temporal arteries of patients with giant cell arteritis (GCA). Representative photomicrographs showing 5 - $\mu \mathrm{m}$-thick paraffin-embedded sections of artery biopsy specimens obtained from controls (A) and patients with GCA (B-F), stained for IL-33. IL-33 expression was observed in inflamed arteries displaying small vessel vasculitis (SVV) (B) and transmural inflammation (C). In SVV IL-33 expression was observed among infiltrating mononuclear cells (arrows) and smooth vascular muscle cells (B). In arteries displaying transmural inflammation IL-33 expression was observed among infiltrating inflammatory mononuclear cells (D) and giant cells scattered through the inflamed wall of inflamed arteries (E). Microvessels distributed through the inflammatory infiltrates also showed intense IL-33 positivity (arrows) (F). (A, original magnification $\times 100$; $B-C$, original magnification $\times 250$; $D-F$, original magnification $\times 400$ ). 


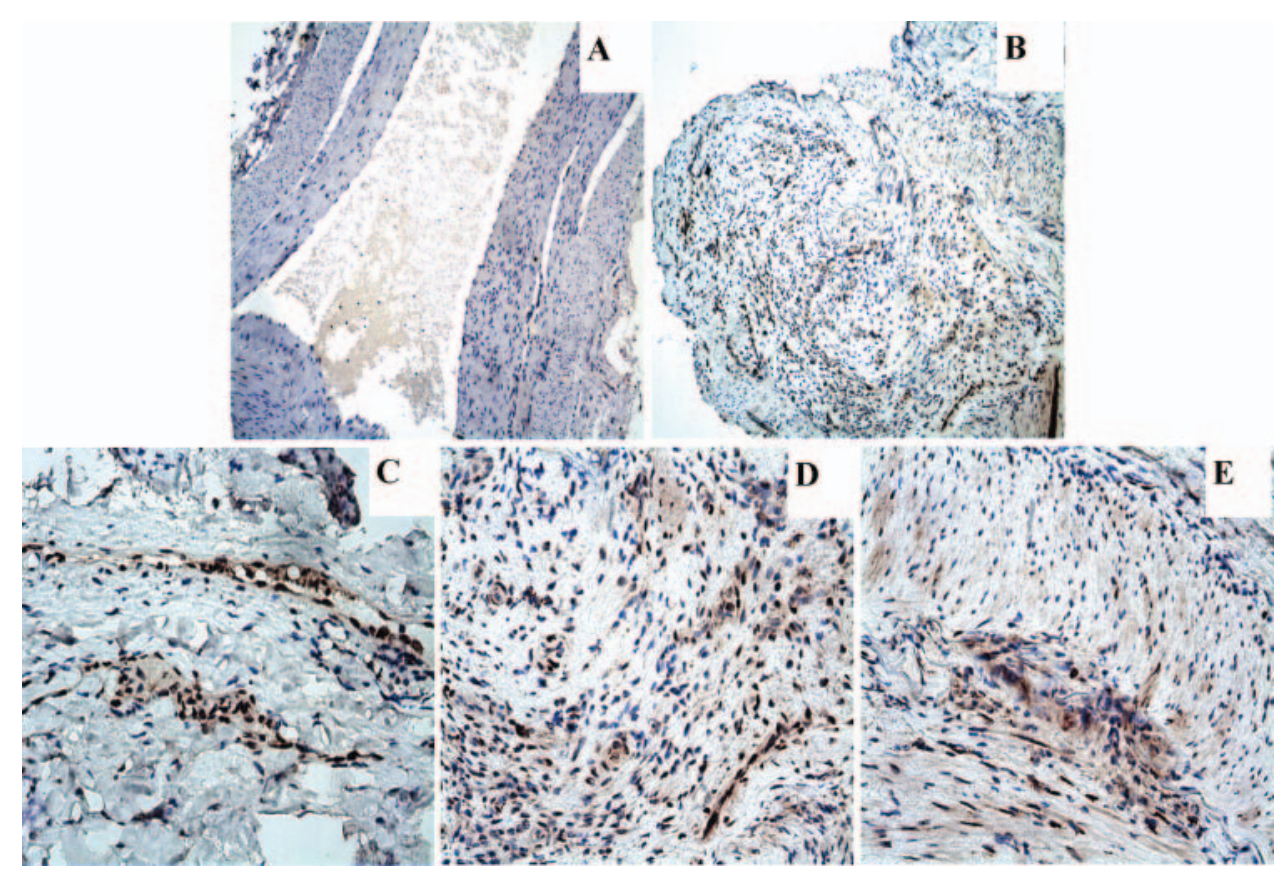

Figure 2 ST2 expression in temporal arteries of patients with giant cell arteritis (GCA). Representative photomicrographs showing 5- $\mu \mathrm{m}$-thick paraffin-embedded sections of artery biopsy specimens obtained from controls (A) and patients with GCA (B-E), stained for ST2. Abundant ST2 expression was observed in microvessels distributed through the inflammatory infiltrates (B-C). ST2 expression was also observed in infiltrating inflammatory mononuclear cells $(D)$ and some giant cells scattered through the inflamed wall of inflamed arteries $(E)$. ( $A-B$, original magnification $\times 100$; C-E, original magnification $\times 250$ ).

protein expression. Increased expression of p-STAT6 was confirmed in the inflamed arteries of GCA patients with transmural inflammation by immunohistochemical analysis (figure 4E-F).

\section{Macrophages in the arteries of GCA patients}

As IL-33 has been reported to act on naive macrophages inducing a $\mathrm{M} 1$ phenotype but also enhancing the differentiation of alternatively activated macrophages (M2), we evaluated the M1 and M2 macrophages in the inflamed arteries of GCA patients. Inducible nitric oxide synthase and CD163 were used to characterise M1 and M2-polarised macrophages, respectively.

In our study a significantly increased expression of IFN- $\gamma$ (fivefold increase, $\mathrm{p}<0.001$, data not shown) and a concomitant

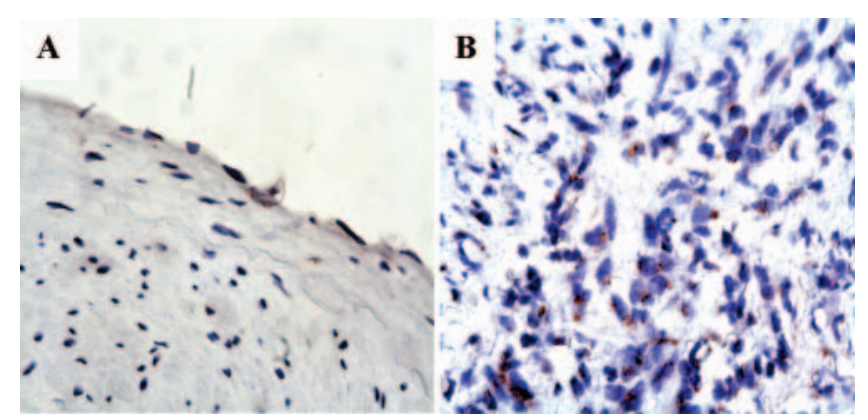

Figure 3 Perivascular IL-1 receptor-associated kinase 1 ( $p$-IRAK1) expression in temporal arteries of patients with giant cell arteritis (GCA). Representative photomicrographs showing 5 - $\mu \mathrm{m}$-thick paraffin-embedded sections of artery biopsy specimens obtained from controls (A) and patients with GCA (B), stained for p-IRAK1. Abundant p-IRAK1 expression was prevalently observed in mononuclear cells scattered through the wall of inflamed GCA arteries $(A-B$, original magnification $\times 250$ ). expansion of M1 macrophages (figure 5) was evident in both the arteries with transmural inflammation (figure 5C) and SVV (figure 5E), suggesting the occurrence in inflamed arteries of a Th1-mediated macrophage activation.

Interestingly, M2 macrophages were also significantly expanded in the inflamed arteries of GCA patients (figure 5D-F) and their presence was significantly correlated with the levels of IL-33 messenger RNA $\left(r^{2}=0.6652, p=0.002\right)$ (data not shown). As other Th2 cytokines were virtually absent, we argue that IL-33 could be responsible for the M2 polarisation observed in GCA patients.

Glucocorticoid therapy modulates IL-33 expression

To analyse the effect of glucocorticoid therapy on IL-33 expression we cross-sectionally compared the mRNA levels and protein expression in arteries from active versus glucocorticoidtreated GCA patients. As shown in figure 6A, in glucocorticoidtreated arteries the expression of IL-33 mRNA was similar to control arteries. Accordingly, the expression of IL-33 at protein level and the numbers of IL- $33^{+}$cells were reduced in glucocorticoid-treated arteries when compared to untreated samples (figure 6B-C).

\section{DISCUSSION}

GCA is a vasculitis of large and medium size vessels whose pathogenesis is actually unknown. A key role in the pathogenesis seems to be played by endothelial cells, which are essential in the induction and perpetuation of the inflammatory process. ${ }^{1} 5$ In GCA, the endothelial cells primarily involved appear to be those of the adventitial vasa vasorum, particularly in early infiltration of the artery wall ${ }^{25}$ and of the newly formed vessels. It is, in fact, at this level that overexpression of adhesion molecules and pro-inflammatory cytokines occurs. ${ }^{3} 26$ In GCA inflammation and angiogenesis seems to be closely associated. $^{26}{ }^{27}$ Interplay between inflammation and 
IL-33

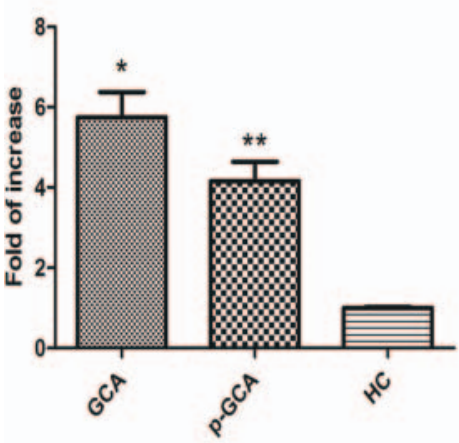

D

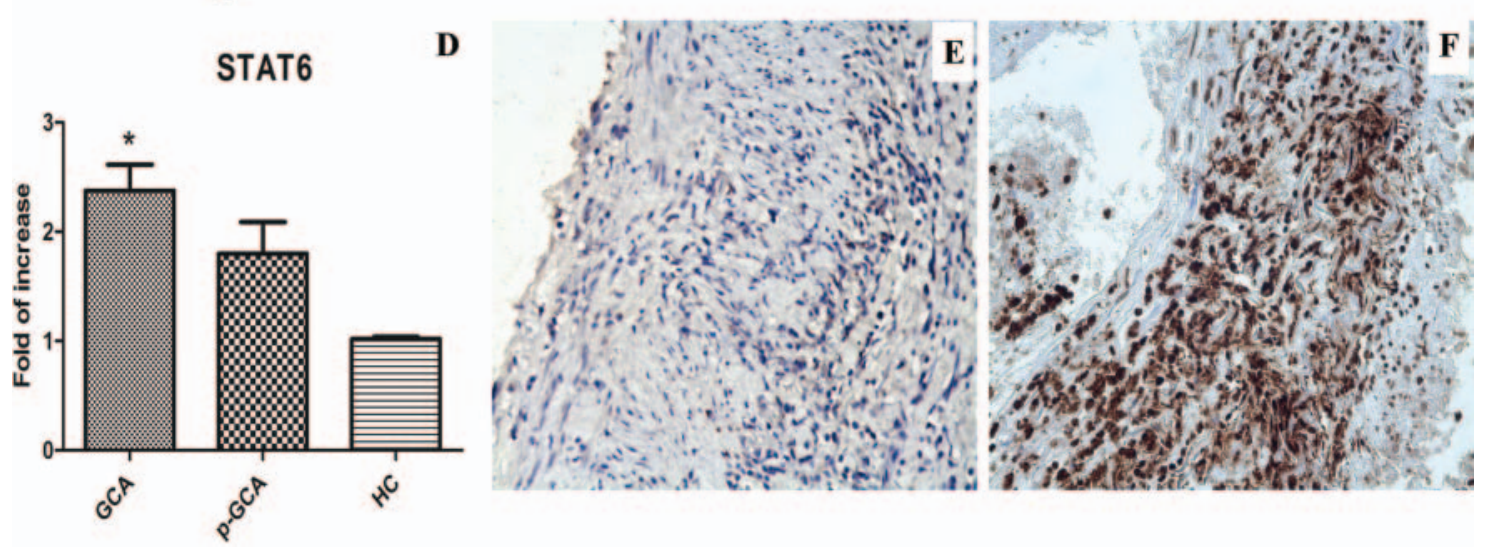

$\mathbf{A}$

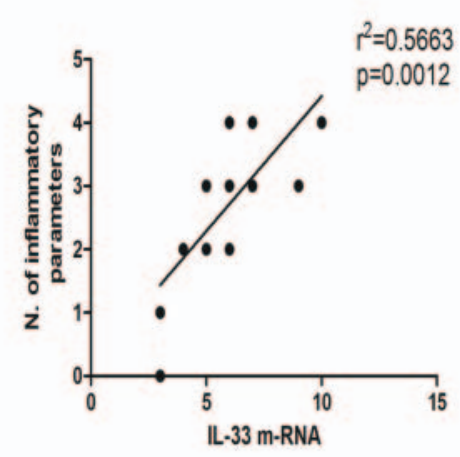

B

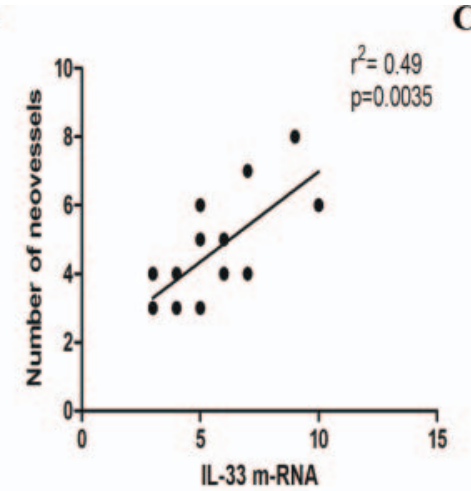

C

Figure 4 Interleukin (IL)-33 and STAT6 gene expression in temporal arteries from patients and controls (HC). Relative mRNA quantification of IL-33 (A) and STAT6 (D) was assessed by quantitative reverse transcriptase PCR in arterial biopsy specimens from patients with transmural (GCA) and perivascular inflammation ( $p$-GCA) and controls. A, D, data are shown as mean (SD). ${ }^{*} p<0.001 ;{ }^{* *} p<0.05$. IL-33 mRNA expression was correlated with the numbers of inflammatory parameters (B) and neovessels $(C)$. E-F, Representative photomicrographs showing 5 - $\mu$ m-thick paraffin-embedded sections of artery biopsies obtained from controls $(E)$ and patients with GCA $(F)$, stained for $p$-STAT-6 $(E-F$, original magnification $\times 250)$.

angiogenesis is largely mediated by cytokines, chemokines and growth factors, and some of these molecules, including IL-6 and vascular endothelial growth factor, have been demonstrated to be increased in GCA. ${ }^{28}$

In the present study we demonstrated an intense expression of IFN- $\gamma$, IL-33, ST2 and STAT6 in the inflamed arteries of GCA patients. The increased expression of IL-33 and its receptor ST2 was mainly observed in endothelial cells of newly formed vessels. Interestingly, IL-33 expression was correlated with the numbers of inflammatory parameters suggesting a participation of IL-33 as a pro-inflammatory cytokine. Both M1 and M2 macrophages were also significantly increased in the inflamed arteries.

IL-33 is a member of the IL-1 cytokine family. ${ }^{6}$ IL-33 is constitutively expressed by endothelial and epithelial cells and is released by these cells following necrotic cell death; for this reason this cytokine has been referred to as an alarmin. ${ }^{8} 1011$ In GCA arteries, IL-33 expression was also observed in vascular smooth muscle cells of the inflamed arteries with adventitial inflammation. It has been shown that vascular smooth muscle cells are exposed to oxidative damage $e^{29} 30$ and IL-33 expression by vascular smooth muscle cells could suggest an 'alarmin' function.

IL-33 is a nuclear protein that is also released into the extracellular space, thus acting as a dual-function molecule. ${ }^{6}$ Extracellular IL-33 binds to the cell-surface receptor ST2, leading to the activation of intracellular signalling pathways similar to those used by IL-1. ST2 is expressed mainly in mast cells, macrophages and Th2 cells. ${ }^{13}{ }^{31}$ ST2 has also been demonstrated to be highly expressed in freshly isolated human umbilical vein endothelial cells, and upregulated by the pro-inflammatory cytokines TNF $\alpha$, IL- $1 \alpha$ and IL-1. ${ }^{7}$ IL-33 has been demonstrated to promote angiogenesis and vascular leakage. ${ }^{12}$ Our demonstration of IL-33 and ST2 intense endothelial positivity together with the positive correlation observed between IL-33 and the numbers of neovessels suggests a role of IL-33 in the pathogenesis of angiogenesis-dependent inflammation in GCA. Upon stimulation of ST2/IL-1R, IRAK1, one of the two putative serine-threonine kinases, undergoes phosphorylation following activation. ${ }^{32}$ The increased expression of p-IRAK1 observed in the inflamed arteries could support the functional relevance of IL-33 and ST2 expression. In this regard the observation of a significant reduction of IL-33 in glucocorticoid-treated patients appears to be of interest and suggests that the efficacy of glucocorticoids on the manifestations of GCA and tissue composition of the inflammatory infiltrates could be at least partly related to the suppression of the IL-33 axis.

Through ST2 interactions IL-33 induces helper T cells, mast cells, eosinophils and basophils to produce type 2 cytokines. In our study, however, IL-33 overexpression was not accompanied by an increased expression of Th2-related cytokines such as IL-4, IL-5, IL-25. The number of samples tested was, however, too small really to confirm this observation.

The Th2-related transcription factor STAT6 was also significantly upregulated at both mRNA and protein levels in the transmural inflamed arteries. Beyond the role of STAT6 in inducing Th2 polarisation, STAT6 has been shown also to be 

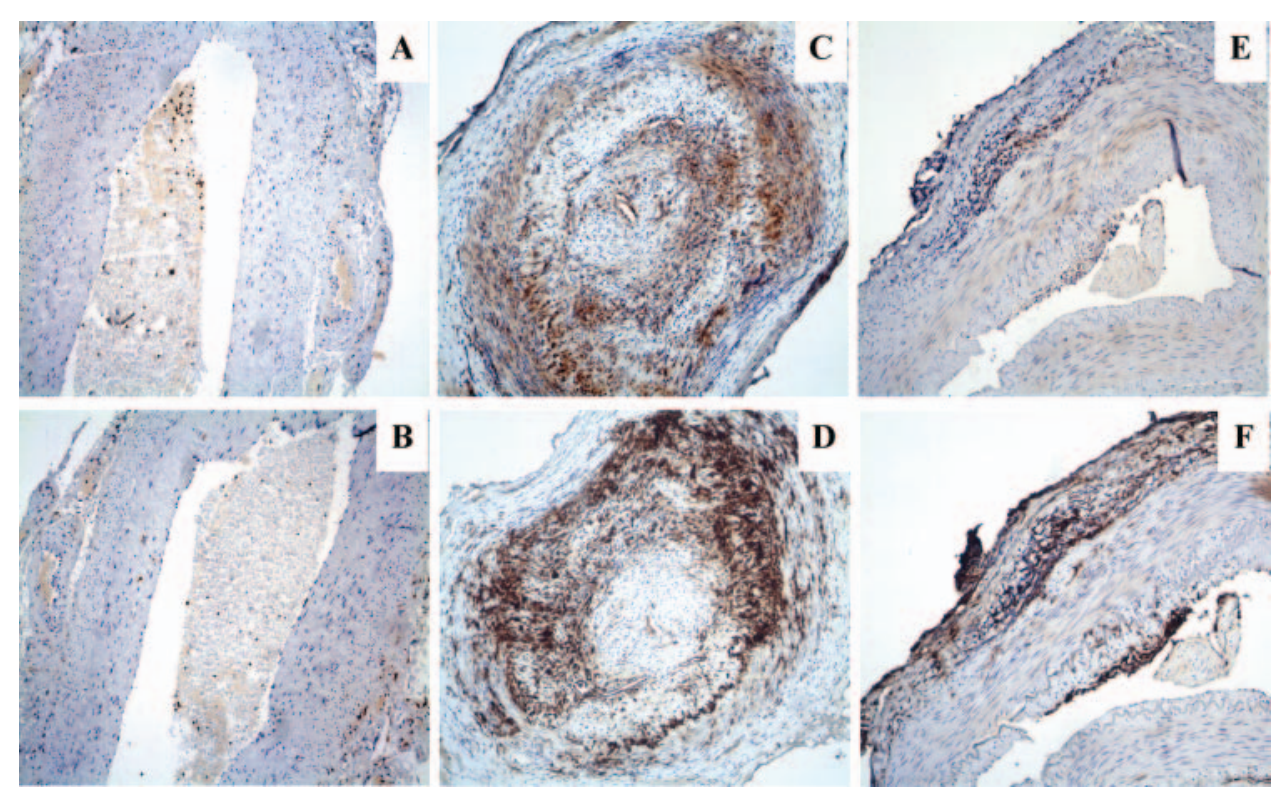

Figure $5 \quad \mathrm{M} 1$ and $\mathrm{M} 2$ macrophages in the temporal arteries of patients with giant cell arteritis (GCA). Representative photomicrographs showing 5 - $\mu$ m-thick paraffin-embedded sections of artery biopsy specimens obtained from controls $(A-B)$ and patients with GCA displaying transmural inflammation and small vessel vasculitis (SVV) (C-F), stained for inducible nitric oxide synthase (iNOS) (a marker of M1 polarisation) and CD163 (a marker of M2 polarisation). A-B, control arteries stained for iNOS (A) and CD163 (B). iNOS ${ }^{+}$(C) and CD163 ${ }^{+}$(D) macrophages were abundantly expressed in the wall of inflamed arteries with transmural inflammation. iNOS ${ }^{+}(E)$ and $C D 163(F)$ macrophages in the wall of inflamed arteries with SVV. (A-F, original magnification $\times 100)$.

involved in the signalling of IL-27, a Th1 cytokine that we have recently demonstrated to be increased in inflamed arteries ${ }^{3}$ suggesting a potential role for STAT6 independent of the Th2 response in GCA.

IL-33 is a crucial amplifier of mucosal and systemic innate immune responses potentiating lipopolysaccharide-mediated cytokine production by macrophages. ${ }^{33}$ In this regard we investigated macrophage polarisation in GCA. Macrophages are routinely categorised into two main phenotypic subtypes based on gene expression induced in response to cytokine and pathogenderived stimulation, ${ }^{16}$ the classically activated macrophages (M1 macrophages) and the alternatively activated macrophages (M2 macrophages). Human macrophages express ST2 and respond to the presence of IL-33 in a different manner depending on the activation status of these cells. ${ }^{16}$ When added to M1 or M2 polarising or polarised conditions, IL-33 promoted the expression of $\mathrm{M} 2$ chemokine markers. ${ }^{13}$ In our study both M1 and M2-polarised macrophages were significantly increased. M1 macrophage polarisation was accompanied by a significantly increased expression of IFN- $\gamma$. The increased number of M2 macrophages was correlated with the levels of IL-33, suggesting that M2 polarisation may be attributable to a direct effect of IL-33 on naive macrophages.

In conclusion, this is the first study demonstrating that the expression of IL-33 and its receptor ST2 is increased in the inflamed arteries of untreated GCA patients and is suppressed in glucocorticoid-treated patients. IL-33 in the inflamed arteries could act earlier in artery inflammation promoting angiogenesis, vascular leakage and tissue infiltration by inflammatory cells. IL-33 may also modulate the innate immune responses through the regulation of macrophage effector functions. Finally, the increased M2 response we observed in this study may suggest that this specific macrophage activation status may play a role in the pathogenesis of GCA.

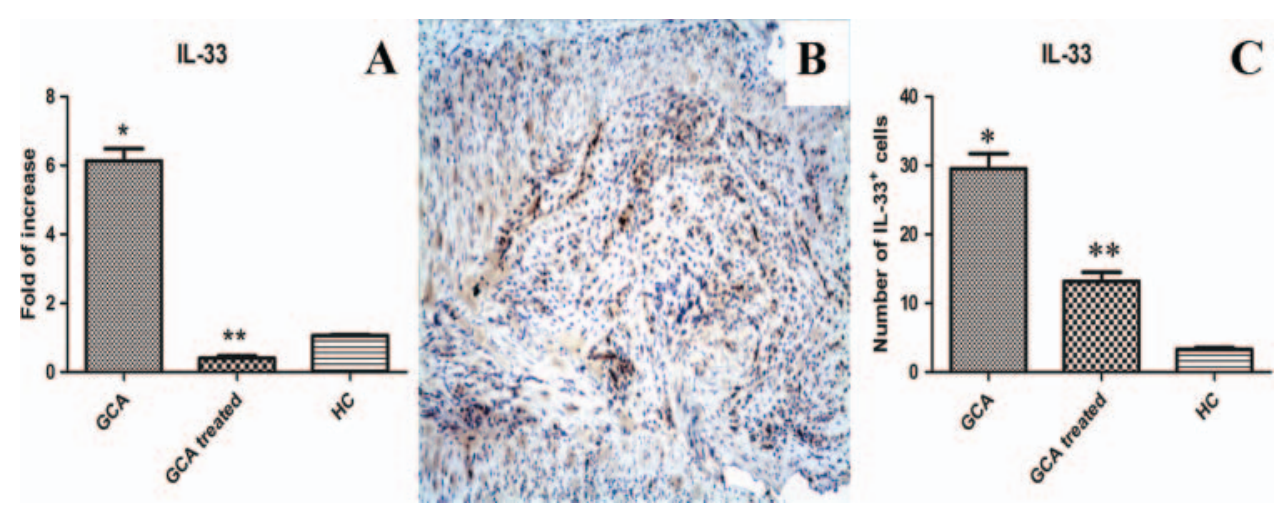

Figure 6 Interleukin (IL)-33 expression in temporal arteries from glucocorticoid-treated patients. (A) Relative mRNA quantification of IL-33 assessed by quantitative reverse transcriptase PCR (data are shown as mean (SD); ${ }^{*} p<0.001 ;{ }^{* *} p<0.05$ ). (B) Representative photomicrograph showing $5 \mu \mathrm{m}$-thick paraffin-embedded sections of artery biopsy specimens obtained from glucocorticoid-treated patient stained for IL-33. (C) Quantification of $\mathrm{IL}-33^{+}$cells in artery samples from steroids untreated and treated patients and controls (HC). Data are shown as mean $(\mathrm{SD}) .{ }^{*} \mathrm{p}<0.0001 ;{ }^{* *} \mathrm{p}<0.05$. 
Contributors Study conception and design: FC, RA, AR, CS, GT. Acquisition of data: all authors. Analysis and interpretation of data: FC, RA, AR, CS, GT. Writing of paper: FC, RA, CS, GT. CS and GT shared co-senior authorship.

Funding This study was supported by a grant from the Ministero della Università e della Ricerca Scientifica of Italy.

Competing interests None.

Ethics approval The study was approved by the ethics committee of local institutions.

Patient consent Obtained.

Provenance and peer review Not commissioned; externally peer reviewed.

\section{REFERENCES}

1. Salvarani C, Cantini F, Hunder GG. Polymyalgia rheumatica and giant-cell arteritis. Lancet 2008:372:234-45.

2. Álvarez Rodríguez L, López-Hoyos M, Mata C, et al. Expression and function of toll-like receptors in peripheral blood mononuclear cells of patients with polymyalgia rheumatica and giant cell arteritis. Ann Rheum Dis 2011;70:1677-83.

3. Ciccia F, Alessandro R, Rizzo A, et al. Expression of interleukin-32 in the inflamed arteries of patients with giant cell arteritis. Arthritis Rheum 2011. 63:2097-104.

4. Deng J, Younge BR, Olshen RA, et al. Th17 and Th1 T-cell responses in giant cell arteritis. Circulation 2010:121:906-15.

5. Weyand CM, Goronzy JJ. Pathogenesis of medium- and large-vessel vasculitis. Z Rheumatol 2009;68:100-7

6. Carriere V, Roussel L, Ortega N, et al. IL-33, the IL-1-like cytokine ligand for ST2 receptor, is a chromatin-associated nuclear factor in vivo. Proc Natl Acad Sci U S A 2007;104:282-7.

7. Demyanets S, Konya V, Kastl SP, et al. Interleukin-33 induces expression of adhesion molecules and inflammatory activation in human endothelial cells and in human atherosclerotic plaques. Arterioscler Thromb Vasc Biol 2011;31:2080-9.

8. Küchler AM, Pollheimer J, Balogh J, et al. Nuclear interleukin-33 is generally expressed in resting endothelium but rapidly lost upon angiogenic or proinflammatory activation. Am J Pathol 2008;173:1229-42.

9. Palmer G, Gabay C. Interleukin-33 biology with potential insights into human diseases. Nat Rev Rheumatol 2011;7:321-9.

10. Haraldsen G, Balogh J, Pollheimer JS, et al. Interleukin-33 — cytokine of dual function or novel alarmin? Trends Immunol 2009;30:227-33.

11. Moussion C, Ortega N, Girard JP. The IL-1-like cytokine IL-33 is constitutively expressed in the nucleus of endothelial cells and epithelial cells in vivo: a novel 'alarmin'? PLoS One 2008;3:e3331.

12. Choi YS, Choi HJ, Min JK, et al. Interleukin-33 induces angiogenesis and vascular permeability through ST2/TRAF6-mediated endothelial nitric oxide production. Blood 2009;114:3117-26.

13. Kurowska-Stolarska M, Stolarski B, Kewin P, et al. IL-33 amplifies the polarization of alternatively activated macrophages that contribute to airway inflammation. $\mathrm{J}$ Immunol 2009;183:6469-77.

14. Gordon S, Taylor PR. Monocyte and macrophage heterogeneity. Nat Rev Immunol 2005:5:953-64.

15. Gordon S, Martinez FO. Alternative activation of macrophages: mechanism and functions. Immunity 2010;32:593-604.
16. Murray PJ, Wynn TA. Protective and pathogenic functions of macrophage subsets. Nat Rev Immunol 2011;11:723-37.

17. Matsuyama $\mathbf{Y}, 0$ kazaki $\mathrm{H}$, Tamemoto $\mathrm{H}$, et al. Increased levels of interleukin 33 in sera and synovial fluid from patients with active rheumatoid arthritis. J Rheumatol 2010;37:18-25

18. Mu R, Huang HO, Li YH, et al. Elevated serum interleukin 33 is associated with autoantibody production in patients with rheumatoid arthritis. J Rheumatol 2010;37:2006-13.

19. Palmer G, Talabot-Ayer D, Lamacchia C, et al. Inhibition of interleukin-33 signaling attenuates the severity of experimental arthritis. Arthritis Rheum 2009;60: 738-49.

20. Sponheim J, Pollheimer J, Olsen T, et al. Inflammatory bowel disease-associated interleukin-33 is preferentially expressed in ulceration-associated myofibroblasts. Am J Pathol 2010;177:2804-15.

21. Theoharides TC, Zhang B, Kempuraj D, et al. IL-33 augments substance p-induced VEGF secretion from human mast cells and is increased in psoriatic skin. Proc Natl Acad Sci U S A 2010;107:4448-53.

22. Hunder GG, Bloch DA, Michel BA, et al. The American College of Rheumatology 1990 criteria for the classification of giant cell arteritis. Arthritis Rheum 1990;33:1122-8.

23. Restuccia G, Cavazza A, Boiardi L, et al. Small-vessel vasculitis surrounding an uninflamed temporal artery and isolated vasa vasorum vasculits of the temporal artery: two subsets of giant cell arteritis. Arthritis Rheum 2011;64:549-56.

24. Schmitz J, Owyang A, Oldham E, et al. IL-33, an interleukin-1-like cytokine that signals via the IL-1 receptor-related protein ST2 and induces T helper type 2-associated cytokines. Immunity 2005;23:479-90.

25. Foell D, Hernández-Rodríguez J, Sánchez M, et al. Early recruitment of phagocytes contributes to the vascular inflammation of giant cell arteritis. J Pathol 2004;204:311-16.

26. Cid MC, Cebrián M, Font $\mathrm{C}$, et al. Cell adhesion molecules in the development of inflammatory infiltrates in giant cell arteritis: inflammation-induced angiogenesis as the preferential site of leukocyte-endothelial cell interactions. Arthritis Rheum 2000:43:184-94.

27. Kaiser M, Younge B, Björnsson J, et al. Formation of new vasa vasorum in vasculitis. Production of angiogenic cytokines by multinucleated giant cells. Am J Pathol 1999;155:765-74.

28. Hernández-Rodríguez J, Segarra M, Vilardell C, et al. Elevated production of interleukin-6 is associated with a lower incidence of disease-related ischemic events in patients with giant-cell arteritis: angiogenic activity of interleukin-6 as a potential protective mechanism. Circulation 2003;107:2428-34.

29. Rittner HL, Hafner V, Klimiuk PA, et al. Aldose reductase functions as a detoxification system for lipid peroxidation products in vasculitis. J Clin Invest 1999;103:1007-13.

30. Rittner HL, Kaiser M, Brack A, et al. Tissue-destructive macrophages in giant cell arteritis. Circ Res 1999;84:1050-8.

31. Xu D, Jiang HR, Kewin P, et al. IL-33 exacerbates antigen-induced arthritis by activating mast cells. Proc Natl Acad Sci U S A 2008;105:10913-18.

32. Gottipati S, Rao NL, Fung-Leung WP. IRAK1: a critical signaling mediator of innate immunity. Cell Signal 2008;20:269-76.

33. Ohno T, Oboki K, Morita H, et al. Paracrine IL-33 stimulation enhances lipopolysaccharide-mediated macrophage activation. PLoS One 2011; 6:e18404. 


\section{$\Delta \mathrm{IL}-33$ is overexpressed in the inflamed arteries of patients with giant cell arteritis}

Francesco Ciccia, Riccardo Alessandro, Aroldo Rizzo, Stefania Raimondo, AnnaRita Giardina, Francesca Raiata, Luigi Boiardi, Alberto Cavazza, Giuliana Guggino, Giacomo De Leo, Carlo Salvarani and Giovanni Triolo

Ann Rheum Dis 2013 72: 258-264 originally published online August 7, 2012

doi: 10.1136/annrheumdis-2012-201309

Updated information and services can be found at:

http://ard.bmj.com/content/72/2/258

References

Email alerting service
These include:

This article cites 33 articles, 12 of which you can access for free at: http://ard.bmj.com/content/72/2/258\#BIBL

Receive free email alerts when new articles cite this article. Sign up in the box at the top right corner of the online article.

ErrataAn erratum has been published regarding this article. Please see next page or:

http://ard.bmj.com/content/74/5/920.full.pdf

Topic Articles on similar topics can be found in the following collections

Collections

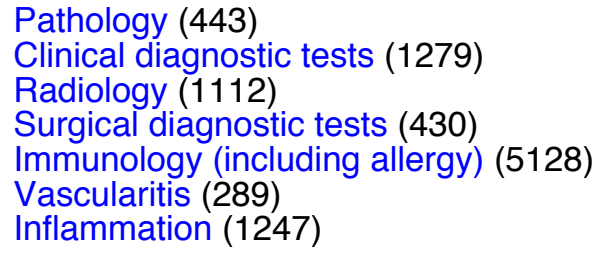

\section{Notes}

To request permissions go to:

http://group.bmj.com/group/rights-licensing/permissions

To order reprints go to:

http://journals.bmj.com/cgi/reprintform

To subscribe to BMJ go to:

http://group.bmj.com/subscribe/ 


\section{Correction}

Ciccia F, Alessandro R, Rizzo A, et al. IL-33 is overexpressed in the inflamed arteries of patients with giant cell arteritis. Ann Rheum Dis 2013;72:258-64. Images in figures 1F and 2C of this paper were identical. The correct figure 1 is given below.
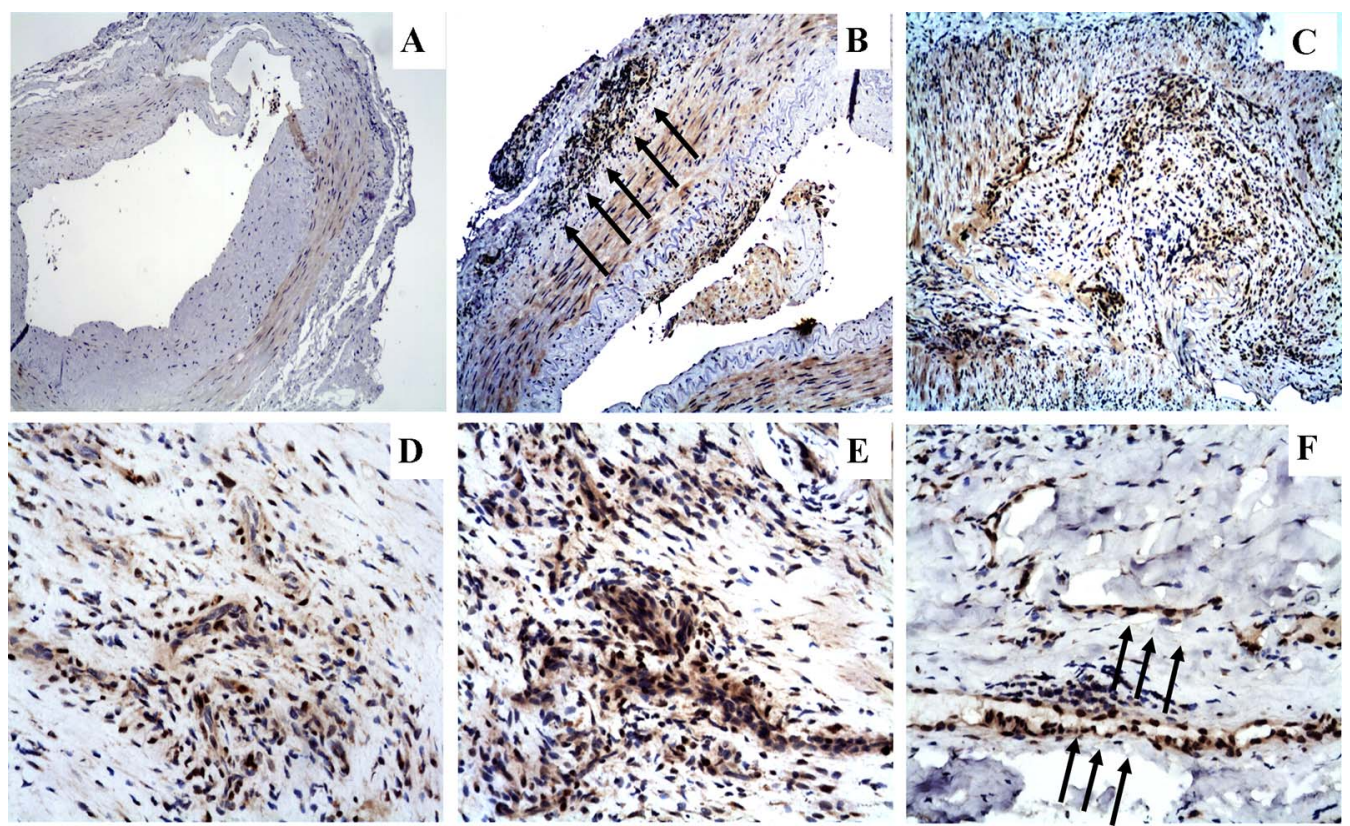

Figure 1 Interleukin (IL)-33 expression in temporal arteries of patients with giant cell arteritis (GCA). Representative photomicrographs showing $5-\mu \mathrm{m}$-thick paraffin-embedded sections of artery biopsy specimens obtained from controls (A) and patients with GCA (B-F), stained for IL-33. IL-33 expression was observed in inflamed arteries displaying small vessel vasculitis (SVV) (B) and transmural inflammation (C). In SVV IL-33 expression was observed among infiltrating mononuclear cells (arrows) and smooth vascular muscle cells (B). In arteries displaying transmural inflammation IL-33 expression was observed among infiltrating inflammatory mononuclear cells (D) and giant cells scattered through the inflamed wall of inflamed arteries (E). Microvessels distributed through the inflammatory infiltrates also showed intense IL-33 positivity (arrows) (F). (A, original magnification $\times 100$; $B-C$, original magnification $\times 250$; $D-F$, original magnification $\times 400$ ).

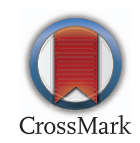

Ann Rheum Dis 2015;74:920. doi:10.1136/annrheumdis-2012-201309corr1 